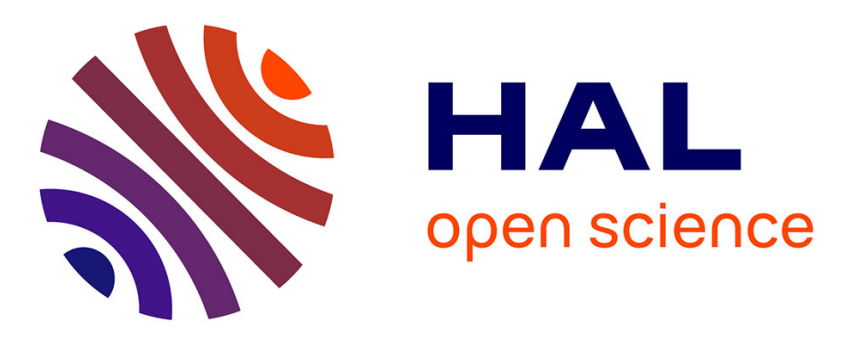

\title{
Model reduction based on sparse identification techniques for induction machines: Towards the real time and accuracy-guaranteed simulation of faulty induction machines
}

Angel Sapena-Bano, Francisco Chinesta, Rubén Puche-Panadero, J.

Martinez-Roman, Manuel Pineda-Sánchez

\section{To cite this version:}

Angel Sapena-Bano, Francisco Chinesta, Rubén Puche-Panadero, J. Martinez-Roman, Manuel PinedaSánchez. Model reduction based on sparse identification techniques for induction machines: Towards the real time and accuracy-guaranteed simulation of faulty induction machines. International Journal of Electrical Power \& Energy Systems, 2021, 125, pp.106417. 10.1016/j.ijepes.2020.106417 . hal03295435

\section{HAL Id: hal-03295435 \\ https://hal.science/hal-03295435}

Submitted on 22 Jul 2021

HAL is a multi-disciplinary open access archive for the deposit and dissemination of scientific research documents, whether they are published or not. The documents may come from teaching and research institutions in France or abroad, or from public or private research centers.
L'archive ouverte pluridisciplinaire HAL, est destinée au dépôt et à la diffusion de documents scientifiques de niveau recherche, publiés ou non, émanant des établissements d'enseignement et de recherche français ou étrangers, des laboratoires publics ou privés. 


\title{
Model reduction based on sparse identification techniques for induction machines: Towards the real time and accuracy-guaranteed simulation of faulty induction machines
}

\author{
A. Sapena-Bano ${ }^{\text {a,*, F. Chinesta }}{ }^{\text {, }}$, R. Puche-Panadero ${ }^{\text {a }}$, J. Martinez-Roman ${ }^{\text {a }}$, M. Pineda-Sanchez ${ }^{a}$ \\ ${ }^{a}$ Institute for Energy Engineering, Universitat Politècnica de València, Camino. de Vera s/n, 46022 Valencia, Spain \\ ${ }^{\mathrm{b}}$ École Nationale Supérieure des Arts et Métiers, Paris, France
}

A R T I C L E IN F O

\section{Keywords:}

Fault diagnosis

Hardware in the loop system

Induction machines

Model order reduction

Real time simualtion

Sparse identification

\begin{abstract}
A B S T R A C T
The development of condition monitoring (CM) systems of induction machines (IMs) is essential for the industry because the early fault detection would help engineers to optimise maintenance plans. However, the use of several IMs to test and validate the fault diagnosis methods developed requires also costly test benches that, anyway, often face limitations in the range of faults and operating conditions to be tested. To avoid it, the use of accurate models such as those based on finite element method (FEM) would reduce the major drawbacks of test benches but their inability to execute FEM models in real time largely reduces their application in the development of on-line continuous monitoring systems. To alleviate this problem a hybrid FEM-analytical model has been proposed. It uses an analytical model that can be run in real-time in a hardware in the loop (HIL) system, after its parameters have been computed through FEM simulations. In this way, the proposed model provides high accuracy but at the cost of long simulation times and high computational costs (both computing power and memory resources) to compute the IM parameters. This work aims at reducing these drawbacks. In particular, a model based on sparse identification techniques is proposed. The method balances complexity and accuracy by selecting a sparse model that reduces the number of FEM simulations to accurately compute the coupling parameters of an IM model with different fault severity degrees. Particularly, the proposed methodology has been applied to develop models with abnormal eccentricity levels as this fault is related to development of mechanical faults that produce most of IM breakdowns.
\end{abstract}

\section{Introduction}

The CM of IMs has been a subject of eager interest over the last years due to the costly downtimes that an unexpected breakdown can cause [1]. The main sources of failures, about the $40-50 \%$ in large IMs, are related to mechanical faults leading to eccentricity [2] with catastrophic consequences [3]. Therefore, the early detection of the eccentricity fault in IMs would be crucial to adjust maintenance plans and ensure the continuity of the industry operation.

During the latest years the detection of the eccentricity fault in IMs has gathered great efforts from the scientific community. In fact, it has been proposed the acquisition and analysis of a wide variety of physical variables of the IM such as the magnetic stray flux $[4,5]$ or vibrations [6] among others. Nevertheless, their use has several drawbacks. On the one hand, their acquisition requires costly sensors, which are also difficult to install on the IM working in the industry. On the other hand, it is not possible to detect all types of faults through the analysis of these magnitudes [7]. Alternatively, the analysis of the stator current has been widely used for the CM of IMs as it is a magnitude conveying relevant information about the machine condition. It is well-known that each type of fault induces or amplifies a family of harmonic components in the stator current, whose frequencies have already been demonstrated theoretically and validated experimentally. Moreover, it has low requirements on hardware and software for its acquisition and analysis.

The eccentricity fault in an IM can appear in three different forms [8] as shown in Fig. 1: static, dynamic or mixed eccentricity. In the case of static and dynamic eccentricity, the rotor symmetry axis is shifted from the stator centre. In the case of static eccentricity, the rotor rotates around its symmetry axis whereas in the case of dynamic eccentricity not. This leads to different configurations of the air-gap width. In the first case, static eccentricity, there are fixed angular position where the air-gap width is minimum and maximum respectively. On the contrary, 


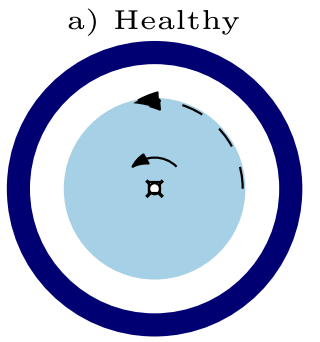

c) Dynamic eccentricity
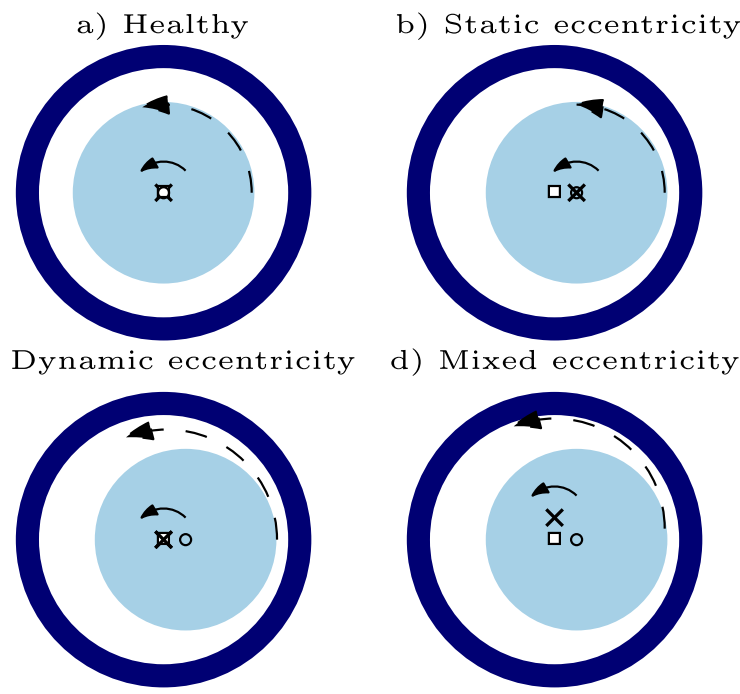

d) Mixed eccentricity

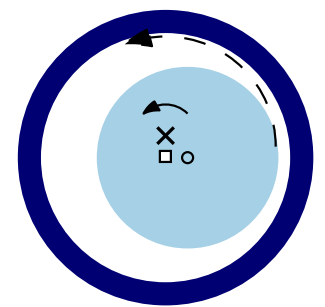

$\square$ Stator centre oRotor centre $\times$ Rotation axe

Fig. 1. Eccentricity types. It can be seen that for static eccentricity the positions for the minimum and maximum airgap widths are fixed regarding the stator for any rotor orientation while for dynamic and mixed eccentricity those positions change as the rotor rotates.

in case of dynamic eccentricity, the position of the minimum and maximum air-gap widths vary as the rotor rotates. The main frequencies due to these type of eccentricities are derived from the general equation to detect the so called principal slot harmonic (PSH) or rotor slot harmonic (RSH) $[8,9]$ :

$f_{h}=\left[\left(k R \pm n_{d}\right) \frac{1-s}{p} \pm v\right] f_{1}$

where $k$ is any positive integer, $R$ are the number of rotor slots, $n_{d}=0$ for static eccentricity or a positive integer for dynamic eccentricity, $s$ is the slip, $p$ is the pole pairs, $v$ is the order of the stator time harmonics and $f_{1}$ is the mains frequency.

Finally, the mixed eccentricity (Fig. 1 d) is a combination of both static and dynamic eccentricities. In this case the low rank fault frequencies that appear are defined by:

$f_{m_{e c c}}=f_{1} \pm k f_{r} \quad k=1,2,3 \ldots$

where $f_{r}$ is the rotor's mechanical angular speed.

A detection of these harmonic components is necessary but not sufficient to perform the diagnosis. IMs are not ideal and always have inherent levels of asymmetries, eccentricities, etc. For example, in the case of static eccentricity, these inherent levels should be lower than $10 \%$ [10], but they may lead to wrong diagnoses. Therefore, the study of the amplitude evolution of these fault harmonic components regarding the fault severity degree will not only allow establishing theoretical thresholds from which detect incipient failures but also reducing misdiagnoses. Besides, the type of machine, the working conditions or the load condition are additional factors influencing the behaviour of the fault harmonic components which should be considered when developing a CM system.

On-line CM systems and artificial intelligence (AI) based fault diagnosis systems would have a major impact in the detection of these faults at early stage. The on-line CM system continuously monitor the machine status obtaining a trend of the eccentricity fault harmonic components to monitor the severity of the upcoming fault. AI based CM systems need to be trained with a large number of signals to learn to classify the machine status (healthy or faulty) and even to determine the severity degree of a given fault. Thus, not only the behaviour but also the reliability of the on-line CM system and the AI based fault diagnosis systems need to be checked with a large number of tests covering a wide variety of scenarios: types of machines, levels of severity of the fault, load conditions, working conditions (steady state, transient), etc.

The ideal is to fulfil these requirements (different IM with different levels of severity of the fault, wide variety of working conditions and load conditions) with IMs working in the industry. However, that would require close and effective collaboration with the industry and, besides, there is a very limited number of IMs that could be running in the industry under faulty conditions. Although laboratory test benches are a good alternative, the tests are limited to the IMs and drives available in the laboratory. Besides, it is very costly because it needs several destructive tests and, from a practical point of view, obtaining several degrees of a given fault or even simultaneous faults is a very challenging task. Therefore, although the experimental validation is inevitable at the last stage of the fully developed $\mathrm{CM}$, during the development stage other less costly alternatives must be considered.

Accurate models could help at reducing these drawbacks: it is possible to obtain different models of several types of IMs, with different levels of severity of a given fault or even with simultaneous faults at much lower cost than using laboratory test benches; moreover, these models enable simulation of IMs under awide variety of working conditions. To achieve these benefits these models must consider the detailed IM structure to obtain simulation results that truly reflect the real-world situations. Besides, these models have to run in real time to properly test on-line condition monitoring systems. However, to achieve these requirements (accuracy and the possibility of running in real-time) in a unique model is very challenging. In the following subsection the main advances in the development of faulty models of IM are reviewed.

\subsection{Faulty IMs models}

Several IMs models have been proposed in the technical literature. The well-known d-q model $[11,12]$ is widely used in order to understand and design vector controlled drives. It is simple to be implemented in a HIL but it does not consider the geometrical complexities, the spatial distribution of the windings (i.e. the space harmonics) [13], the non-linearity of the core materials and it cannot include the effects that a fault introduces in a machine and, thus, it cannot be used for fault diagnosis purposes. To include these features in the model other analytical approaches have been proposed in the technical literature such as the multiple coupled circuit model (MMC) [14], the winding function approach (WFA) [15], the Concordia transformations [16], the use of natural variables [17], the voltage-behind-reactance formulation [18], the magnetic equivalent circuit (MEC) [19] or the sparse identification [20]. Nevertheless, these approaches cannot consider non-ideal conditions and cannot include the effect of the rotor and stator slots in the air-gap magnetic force distribution, specially, when the eccentricity is being modelled [21], as required in faulty IM models. Particularly, the sparse identification is proposed to improve the efficiency of motor control [20] and uses the stator voltages and currents of an induction motor to compute the paramenters of the equivalent circuit. However, it assumes some simplifications such as uniform air-gap width which is enough for motor control but not suitable for fault diagnosis purposes.

On the other hand, FEM models and their accuracy are widely accepted as they usually take into account the geometrical complexities, the spatial distribution of the windings, the non-linearity of the core materials, etc [22]. Unfortunately, time-stepping FEM simulations require high computing power and memory resources. Besides, they take long simulation times (from minutes to days) for short simulation periods. These constrains are even worse with faulty IM models where simplifications to boost the time-stepping FEM simulation, such as the symmetry boundary conditions,can no longer be applied. To sum up, FEM highly increases the accuracy in machine simulation [22], but at 
significant $\mathrm{c}$ omputational $\mathrm{c}$ ost e ven $\mathrm{w}$ ith $\mathrm{m}$ odern $\mathrm{p}$ rocessing power computers [23]. Consequently, the savings in computational effort are crucial in fields where a large number of results are required such as fault diagnosis, either for on-line CM systems or for AI based fault diagnosis systems, motor control optimization, etc.

Hybrid FEM-analytical models have been recently proposed as they are able to run in real time in a HIL simulator and keep good accuracy [24-27]. They are based on the equivalent circuit parameters computation through magneto- static FEM simulations and on using these parameters in the analytical model [27]. However, the hybrid FEManalytical model still has several limitations as the evaluation of each new scenario (fault conditions) requires the full FEM analysis to compute the new coupling parameters with its corresponding long running times and computational effort.

In an attempt to address these drawbacks [28] proposes the sparse subspace learning (SSL) in combination with a hierarchical collocation strategy to compute a low-rank prediction of the parametric solution of the FEM model. In fact, the SSL uses the outputs of a deterministic solver to produce parametric solutions in a multi-level interpolation framework. Thereafter, the deterministic solver uses these predicted solutions as input, as initial guess, to obtain the solution in a new sampling point. In that case, the initial guess is so close to the solution that the iteration time of the solver is drastically reduced or might not even be required to run. From the point of view of the fault diagnosis purposes, this approach does not mean any substantial improvement because it does not reduce the large number of magneto-static FEM simulations required to obtain the coupling parameters [27] for the analytical model. To alleviate this problem the sparse identification was proposed in [29] to obtain a faulty IM model. However, to perform the parameter identification of a new faulty IM model the method requires the input of not only a wide range of fully FEM computed coupling parameters of the same machine with different severity degrees of the fault under study. Therefore, it reduces the computational effort compared with traditional methods, but it still requires a large number of FEM simulations. Besides, it is very limited to be applied to other machines or even to other faults.

To ease the limitations of traditional SSL implementation, and as a novelty, this paper proposes the use of the sparse identification technique aimed at reducing the number of magneto-static FEM simulations. This will avoid the need of a FEM simulation for every new sampling point, as in [28]. Therefore, this paper proposes the sparse identification $t \mathrm{o}$ c ompute the c oupling $\mathrm{p}$ arameters of the faulty IM model based on the results of a very reduced number of magneto-static FEM simulations. This will not only reduce the computational effort but will also guarantee good accuracy of the obtained model.In this paper, the proposed method is applied to obtain models with static eccentricity faults as it is a fault that may lead to catastrophic failures and because it is very difficult to artificially force different degrees of the fault in IM to be used in test benches. These models will provide a better understanding of the physical phenomena while tracking the behaviour of the fault harmonic components, to establish thresholds, etc. Besides, as these models are capable to run in real time under different working conditions (power supply variations, load changes, etc), they will be useful for developing on-line CM systems and to train AI based automatic diagnostic systems.

The paper is structured as follows. In Section 2 the hybrid FEManalytical model is described and the methodology to compute the coupling parameters is introduced showing the main drawbacks of the approach. Section 3 introduces the proposed method to compute the coupling parameters and the main benefits are presented in terms on computing time and memory requirements. The main results are presented in Section 3.3 In this section the coupling parameters computed with the proposed method are compared with those obtained with traditional methods. Moreover, the achieved accuracy is as good as the savings in terms on computing time and memory for data storage. In Section 4 the simulations results in terms of fault diagnosis purposes are presented while in Section 5 the experimental validation is described. Finally, in Section 6 the main conclusions are presented.

\section{Hybrid FEM-analytical model of an IM}

The electromagnetic behavior of a general IM with $M$ stator and $N$ rotor phases can be modelled as [30-32]:

$\left[\begin{array}{c}U_{S_{1}} \\ \vdots \\ U_{S_{M}} \\ U_{r_{1}} \\ \vdots \\ U_{r_{N}}\end{array}\right]=\left[\begin{array}{llllll}R_{S_{1}} & & & & & \\ & \ddots & & & & \\ & & R_{S_{M}} & & & \\ & & & R_{r_{1}} & & \\ & & & & \ddots & \\ & & & & & R_{r_{N}}\end{array}\right]\left[\begin{array}{c}I_{S_{1}} \\ \vdots \\ I_{S_{M}} \\ I_{r_{1}} \\ \vdots \\ I_{r_{N}}\end{array}\right]+\frac{\mathrm{d}[\Psi]}{\mathrm{d} t}$

where the subscripts $s$ and $r$ stand for stator and rotor respectively, [U] is the phase voltage matrix, $[R]$ is the resistances matrix, $[I]$ is the phase current matrix and $\Psi$ stands for the flux linkage that can be computed as:

$\left[\begin{array}{c}\Psi_{s_{1}} \\ \vdots \\ \Psi_{s_{M}} \\ \Psi_{r_{1}} \\ \vdots \\ \Psi_{r_{n}}\end{array}\right]=\left[\begin{array}{ccc|ccc}L_{s_{1} s_{1}} & \ldots & L_{s_{1} s_{M}} & L_{s_{1} r_{1}} & \ldots & L_{s_{1} r_{N}} \\ \vdots & \ddots & & & & \vdots \\ L_{s_{M} s_{1}} & \ldots & L_{s_{M} s_{M}} & L_{s_{M} r_{1}} & \ldots & L_{s_{M} r_{N}} \\ \hline L_{r_{1} s_{1}} & \ldots & L_{r_{1} s_{M}} & L_{r_{1} r_{1}} & \ldots & L_{r_{1} r_{N}} \\ \vdots & & & & \ddots & \vdots \\ L_{r_{N} s_{1}} & \ldots & L_{r_{N} s_{M}} & L_{r_{N} r_{1}} & \ldots & L_{r_{N} r_{N}}\end{array}\right]\left[\begin{array}{c}I_{s_{1}} \\ \vdots \\ I_{s_{M}} \\ I_{r_{1}} \\ \vdots \\ I_{r_{N}}\end{array}\right]$

where $[L]$ is also known as the inductances matrix, that is to say, the coupling parameters between the different electromagnetic circuits inside of an IM. To simplify, (4) can also be expressed as:

$\left[\begin{array}{l}\Psi_{s} \\ \Psi_{r}\end{array}\right]=\left[\begin{array}{l|l}L_{s s} & L_{s r} \\ \hline L_{s r}^{T} & L_{r r}\end{array}\right]\left[\begin{array}{c}I_{s} \\ I_{r}\end{array}\right]$

where $\left[L_{s S}\right],\left[L_{s r}\right]$ and $\left[L_{r r}\right]$ are the coupling parameters between stator phases, between stator and rotor phases and between rotor phases respectively. The electromagnetic torque generated $T_{e}$ by the IM is modelled as:

$T_{e}=\left[I_{s}\right]^{T} \frac{\mathrm{d}\left[L_{s r}\right]}{\mathrm{d} \theta}\left[I_{r}\right]$

and finally, the equation that models the mechanical behaviour is:

$T_{e}-T_{L o a d}=J \frac{\mathrm{d}^{2} \theta}{\mathrm{d} t^{2}}+b \frac{\mathrm{d} \theta}{\mathrm{d} t}=J \alpha+B \omega$

where $T_{\text {Load }}$ is the load torque, $J$ is the inertia constant, $B$ is viscous friction constant, $\alpha$ is the angular acceleration and $\omega$ is the rotational speed. The analytical model of an IM using the system of Eqs. (3) to (7) can be easily implemented in a Simulink model as shown in Fig. 2. The main advantage of this type of model is that can be run in real time in a HIL system. It must be highlighted that all blocks used are standard Simulink blocks except for the "OpComm" block belonging to the HIL library and used to connect the input signals of the model. The model shown in Fig. 2 uses the stator voltages and the load torque as inputs. For instance, to cover a wide variety of industrial scenarios the user can select to power the IM either using direct on-line (DOL) (balanced or unbalanced phase voltages) or through a variable speed drive (VSD) with its usual open/close loop controls. Moreover, during the real-time simulation the user could apply changes in the power supply such as the voltage and/or the reference speed of the VSD. On the other hand, the user can define load torque profiles to simulate industrial processes and/or modify the load torque during the real time simulation. Therefore, it allows to simulate the IM model under a wide variety of working conditions as required for the CM systems development.

In this approach, the key issue is to compute the coupling parameters $[L]$ of the faulty IM model. Specifically, the presence of derivatives in (6) requires an accurate enough computation of the coupling parameters for the reliable identification of different fault severity 


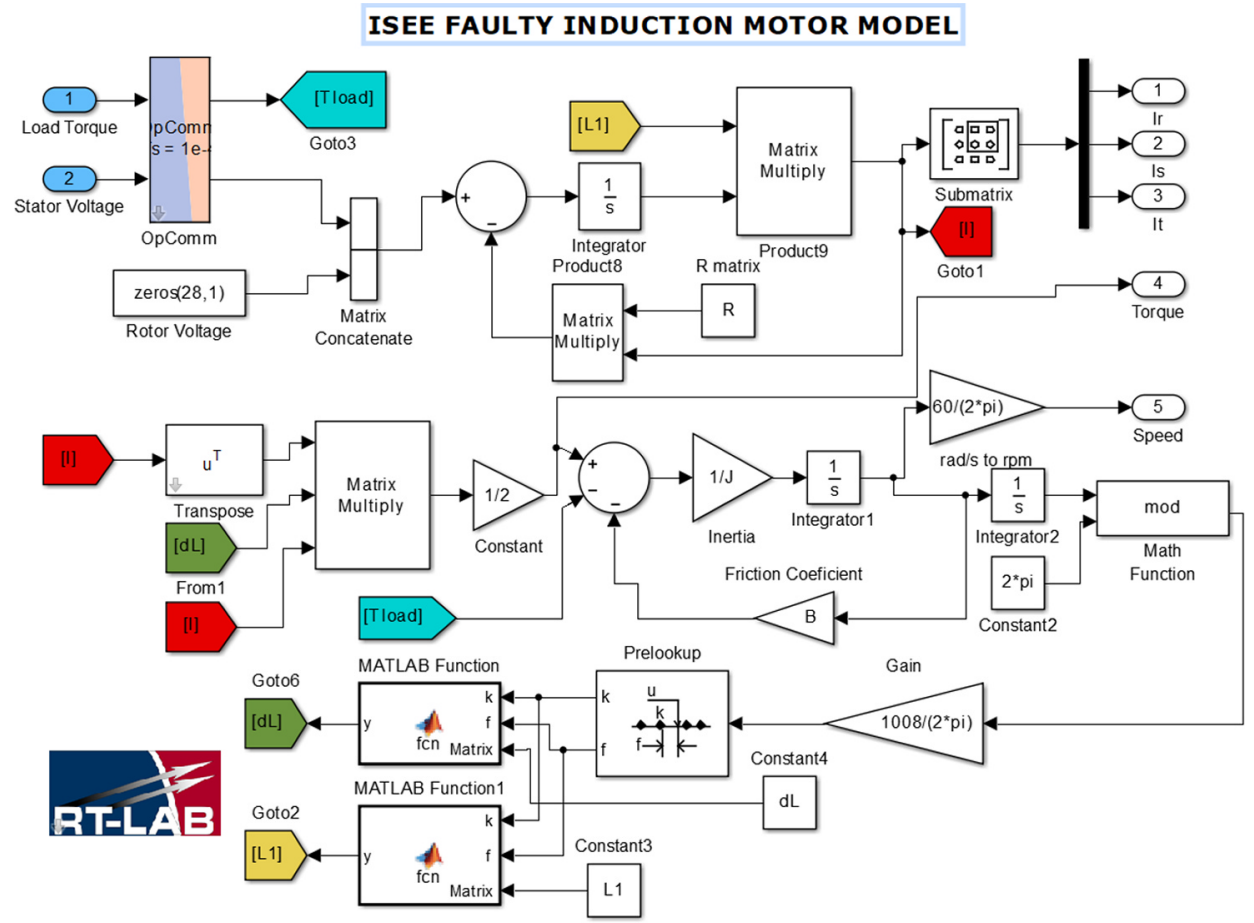

Fig. 2. Analytical model using the system equations (3)-(7) using Simulink blocks ready to be run in a HIL system for real-time simulation.

degrees. As the coupling parameters $[L]$ vary depending on the rotor position, specially in the case of a faulty machine, it is necessary to compute the mutual and self inductance for each rotor position requiring, thus, a large number of magneto-static FEM simulations.

To illustrate the main drawbacks associated with the coupling parameters $[L]$ computation based on FEM software and to introduce and show the main benefits of the proposed method the machine whose main characteristics are shown in Table 1 is used. Given the importance of the static eccentricity fault, this fault has been used to illustrate the method but the same procedure of Sections 2.1 and 3 is also valid for other different types of faults.

\subsection{Methodology to compute the parameters of the IM model using FEM for a generic case}

FEM software allows to create accurate IM models which consider the non-uniform air-gap due to stator and rotor and other asymmetries due to faults. Therefore, in opposition the simulation requirements for healthy machines, the whole geometry of a faulty machine has to be considered in the simulation which results in a much more time-consuming task. Besides, a large number of simulations are required to compute the coupling parameters matrix.

Fig. 3 shows the general diagram to compute the coupling parameters $[L]$ for an IM. The process starts by creating a FEM model of the IM in which the geometry of the machine as well as the specific characteristics of the fault are considered and the rotor is placed in the first position $(q=1)$. Starting from the first stator phase $(m=1)$ each of the

Table 1

Data of the simulated machine.

\begin{tabular}{lclc}
\hline & \multicolumn{2}{c}{ Electrical } & \multicolumn{2}{c}{ Mechanical } \\
\hline Power & $1.1 \mathrm{~kW}$ & Pole pairs & 2 \\
Voltage & $230 / 400 \mathrm{~V}$ & Speed & $1415 \mathrm{rpm}$ \\
Current & $4.4 / 2.55 \mathrm{~A}$ & $\mathrm{~N}^{\circ}$ of rotor bars & 28 \\
Frequency & $50 \mathrm{~Hz}$ & $\mathrm{~N}^{0}$ of stator slots & 36 \\
& & Airgap length & $0.28 \mathrm{~mm}$ \\
& & &
\end{tabular}

$M$ stator phases are fed with $1 \mathrm{~A}$ DC and the magneto-static FEM simulation is performed. With the results it is possible to compute the coupling parameters between stator phases $\left[L_{s s}\right]$ and between stator and rotor phases $\left[L_{s r}\right]$. Usually, in case of cage IM the coupling parameters between stator phases and rotor bars are considered instead of between stator and rotor phases. Subsequently, each of the $N$ rotor phases is fed by 1 A DC, the FEM magneto-static simulation is performed and the coupling parameters between rotor phases (rotor bars in case of cage IM) $\left[L_{r r}\right]$ are computed. The rotor is moved in increments of $r d=2 \pi / K$, where $K$ is the number of positions desired, and the aforementioned process is repeated for each rotor position. Clearly, the larger the $K$ considered the higher the accuracy in the description of the coupling parameters $[L]$ for different rotor positions and the higher the number of FEM simulations required with its corresponding running time, computing power and memory resources. Finally a three dimension $(M+N, M+N, K)$ coupling parameters matrix $[L]$ is obtained, where the third dimension is related to the rotor position $q$. Hence, the coupling parameters matrix $\left[L_{a b q}\right]$ designated from now as $\left[L_{q}\right]$ is the coupling parameter matrix $[L]$ of dimensions $(M+N, M+N)$ corresponding to the rotor position $q$.

The method proposed in this paper assumes linear conditions for the computation of the inductance matrix of the eccentric machine. From a diagnostic point of view, saturation generates fault harmonics which are different from those generated by the eccentricity fault. Indeed, the purpose of the presented approach is to develop an efficient method for computing an inductance matrix that accurately captures the effect of the eccentricity fault. The proposed paper aims at developing an analytical model able to run in real-time with high accuracy (based on FEM results) but a much lower cost. In this case, considering only the linear, incremental problem, balances the results with the computational costs obtaining a reasonably accurate solution for eccentricity fault diagnosis purposes. Nevertheless, if the saturation effects are also to be reproduced, there are several methods presented in the technical literature where the inductance matrix obtained with the proposed approach can be used. One of such methods is the "incremental permeability" solution proposed in [33], which is used in several FEM software packages to save computation time. In this solution, first, the nonlinear 


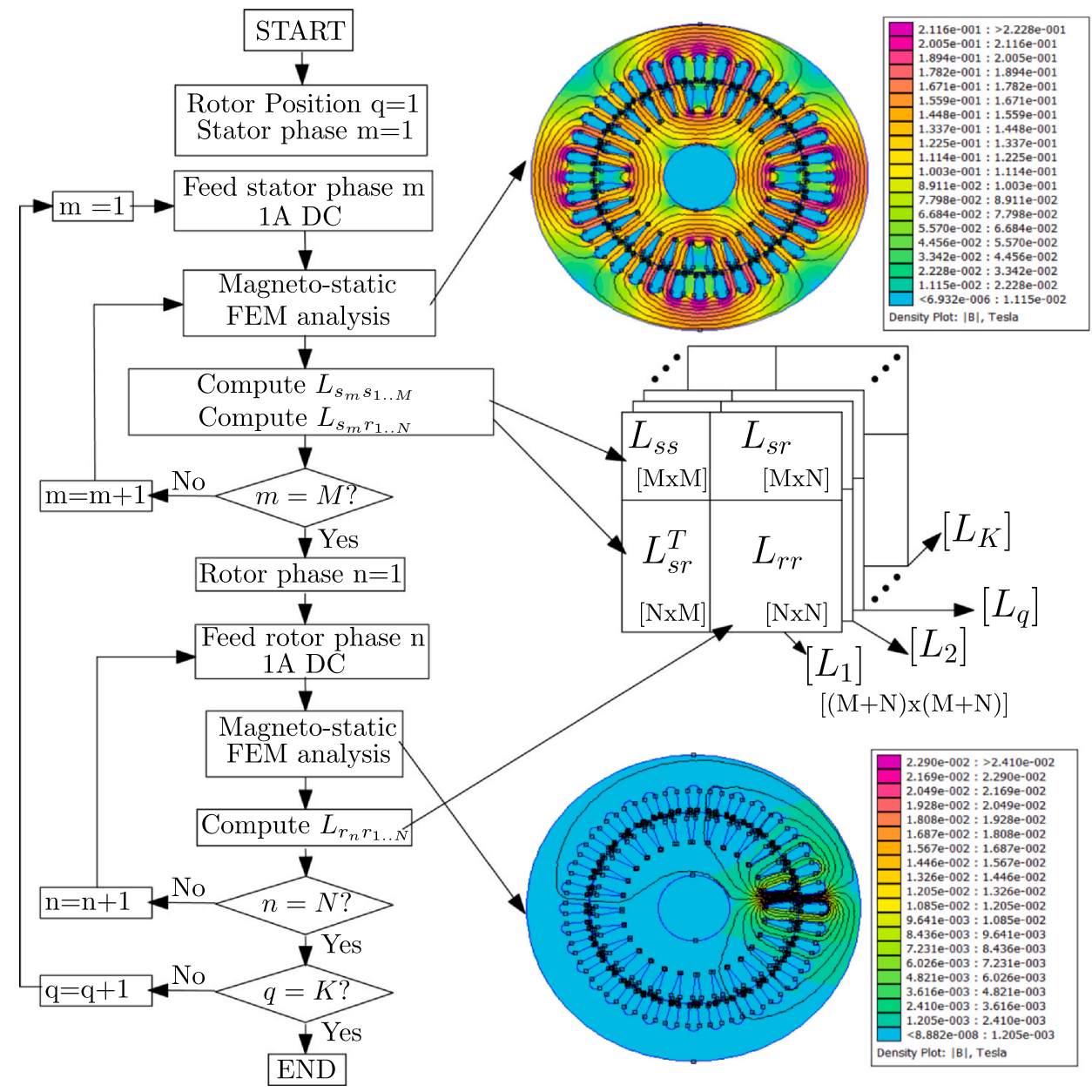

Fig. 3. General diagram of the procedure to compute the coupling parameters $[L]$ of a IM model using FEM software.

problem is solved at a particular nonlinear operating point (particular instantaneous currents, rotor orientation), using a full FEM simulation. Then, a linear, incremental problem based on the incremental permeabilities is solved, by feeding each phase with a current of $1 \mathrm{~A}$ with all other excitations turned off. This linear problem is solved in [33] using again FEM simulations, which could be replaced by the solutions provided by the proposed method, in a much faster way, while keeping their accuracy.

\subsubsection{Particularizing the method to compute the coupling parameters to the IM under study}

To illustrate the cost in terms of computing power, memory resources and processing time, the method introduced in Section 2 and illustrated in the Fig. 3 has been applied to obtain the coupling parameters matrix $[L]$ of the IM whose main characteristics are shown in Table 1. The software used for the FEM simulations is the open source femm 4.2 and the computer used has an intel processor (R) Core (TM) i5-6400 CPU@2.70 GHz 2.20 GHz and 16 GB of RAM memory.

The first step, is to decide the rotor movement steps $(r d=2 \pi / K)$ to be used. This term is related to the accuracy, in terms of rotor position, needed to compute the coupling parameters $[L]$. Usually, the result of multiplying the number of stator slots by the number of rotor bars for cage IM (or rotor slots in case of wound rotor IM) provides a good value of $K$ :

$K=\mathrm{n}^{\mathrm{er}}$ of rotor bars $\times \mathrm{n}^{\mathrm{er}}$ of stator slots $=28 \times 36=1008$

and, therefore $r d=2 \pi / K=2 \pi / 1008=0.00632$ rads. Considering the process shown in Fig. 3 where for the IM studied $K=1008$, a rotor phase is the loop of two adjacent rotor bars $N=28 / 2$ and the number of stator phases is $M=3$, the computation of the coupling parameters matrix $[L]$ in this generic case requires $(M+N) \cdot K=(3+28 / 2) \cdot 1008=17,136$ magneto-static FEM simulations. Taking into account that each magneto-static FEM simulation takes $1 \mathrm{~min}$ and needs $22.5 \mathrm{MB}$ for data storage, the computation of the coupling parameters $[L]$, for one machine and with only one severity degree for a given fault, would require 11 days $21 \mathrm{~h}$ and $36 \mathrm{~min}$ and $376.52 \mathrm{~GB}$ for data storage if all the FEM results need to be saved; if only the coupling parameters $[L]$ are needed each $\left[L_{q}\right]$ (coupling parameters for a rotor position) require $1 \mathrm{kB}$, so that, the memory resources could be reduced to just $1008 \mathrm{kB}$. It must be highlighted that these resources are needed to compute the coupling parameters of just 1 machine and only for 1 severity degree of a given fault whereas to fulfil the requirements for developing on-line CM systems and expert systems a wide variety of models of different machines and with several severity degrees of a given fault are needed.

\subsubsection{Particularizing the method to compute the IM coupling parameters considering the static eccentricity fault}

Sometimes, the particularities of a specific fault enable the use of some simplifications to reduce the computing effort for computing the coupling parameter matrix L. To reproduce the static eccentricity fault the rotor symmetry axis have to be displaced from the stator centre. It allows the definition of different degrees of static eccentricity fault, from $0 \%$ for healthy conditions to $100 \%$ for the maximum displacement of rotor symmetry axis. It results in a faulty IM model where the positions of the maximum and minimum air-gap width with respect to the stator do not depend on the rotor position. Therefore, each rotor phase (rotor bar) will have the same flux linkage but with a certain geometric offset. As a consequence, two main simplifications can be applied; only one rotor phase (or rotor bar) must be fed to compute the coupling 
parameters between rotor phases (or rotor bars) $\left[L_{r r}\right]$; and this bar has to be displaced only along half of all the possible rotor positions, that is to say, between $[0, \pi]$ which, in the case of study, is 504 of the 1008 total positions.

On the other hand, to compute the coupling parameters between stator and stator phases $\left[L_{s s}\right]$ and between stator phases and rotor phases (bars) $\left[L_{s r}\right]$ just the positions of a rotor bar travelling through a stator slot are required. Therefore, the computation of $\left[L_{s s}\right]$ and $\left[L_{s r}\right]$ requires just the simulation of the model placing the rotor in the first 36 of the 1008 positions while feeding, sequentially, each stator phase. It reduces the required FEM simulations to $3 \cdot 36+504=612$ FEM simulations with a computing time of $10 \mathrm{~h}$ and $12 \mathrm{~min}$ and a data storage of 13.45 GB. It is a significant improvement but it does not go far enough to meet the needs of the development of CM systems as they require to test not only a wide variety of IMs but also a wide range of severity degrees of the faults.

\section{Proposed method: sparse identification to compute the parameters of the IM model with similar accuracy to FEM}

\subsection{The Sparse Subspace Learning (SSL) and the Hierarchical Lagrange interpolation (HLI)}

The SSL in combination with a hierarchical Lagrange interpolation (HLI) as polynomial basis introduced in [28] reduces the computing time needed to solve parametric problems in FEM software. It proposes a collocation strategy to reduce the time computing requirements of parametric models. The SSL strategy selects specific sampling points in which the simulation has to be performed; thereafter, the output of the deterministic solver is used to obtain a HLI polynomial basis which allows to compute an approximate low-rank parametric solution at new sampling points. This approximated solution is used to initialize the FEM solver which speeds up the convergence of the iteration process as the predicted solutions are very close to the FEM solution; in fact, in some cases, the iteration process is not even required to be run. In many cases with moderate dimensionality, the iteration process is not needed as the hierarchical predicted solution yields precise enough results for most engineering problems at a reasonable computational costs [34].

\subsection{Sparse identification to compute the coupling parameter matrix}

With the sparse identification strategy proposed in [28] the polynomial basis obtains a prediction of the solutions in the nodes of the FEM model. Although it reduces the computing time for complex models, it keeps the memory requirements for data storage. If we contextualize to compute the coupling parameters of a faulty IM, where a magneto-static FEM simulation takes just $1 \mathrm{~min}$, the main benefits of the sparse identification shall not have a major impact in the overall computation time. Therefore, what is proposed in this paper, is to go one step further by performing the sparse identification to obtain the coupling parameters matrix $[L]$ of a faulty IM. This matrix will be computed with a polynomial basis built from results of a few FEM simulations. Hence, it will reduce not only the computing time but also the memory requirements for data storage.

In this case, for a faulty IM model with a specific degree of static eccentricity fault, the coupling parameters $[L]$ vary depending on the rotor position $\theta$. This paper proposes the SSL strategy to select the rotor positions $\theta$ in the parametric space $\left[\theta_{\min }, \theta_{\max }\right]$ in which the FEM simulations have to be performed. Thereafter, the results, i.e. the coupling parameters for these specific rotor positions, will be used to compute the polynomial basis with which compute the coupling parameters $[L]$ for the remaining rotor positions.

When using polynomial approximation an optimal choice for the sampling is defined by the set of Gauss-Chebyschev-Lobatto (GCL) points:
$\boldsymbol{P}^{(k)} \equiv \begin{cases}\left\{\theta_{\min }, \theta_{\max }\right\} & \text { if } \quad k=0 \\ \left\{\theta_{j}=\left(\frac{\theta_{\min }+\theta_{\max }}{2}\right) \cdot\left(\cos \left(\frac{2 j-1}{2^{k}} \pi\right)+1\right)\right. & \text { if } \quad k>0 \\ \left.\forall j=1, \ldots 2^{k-1}\right\} \cup \mathcal{P}^{(k-1)} & \end{cases}$

where $\boldsymbol{P}^{(k)}$ are the selected points for the hierarchical $k$ level. This means that the corresponding set of points $\mathcal{P}^{(k)}$ for the hierarchical level $k$ has $N^{(k)}$ elements. It implies that each level contains the $N^{(k-1)}$ elements of the previous levels plus the $N^{(k)}-N^{(k-1)}$ additional points [28]. To build the polynomial basis the Lagrange interpolation is considered. Therefore, for a given hierarchical level $k$ and $N^{(k-1)}<j<N^{(k)}$, the HLI polynomial basis is constructed as:

$\mathcal{L}_{j}^{k}(\theta)=\prod_{\theta_{i} \in \mathcal{P}^{(k), i\urcorner=j}} \frac{\theta-\theta_{i}}{\theta_{j}-\theta_{i}}$

and the coupling parameter row $a$ column $b$ for the rotor position $\theta$, in the coupling parameter's matrix with the proposed method $L_{a b}^{H L I}$ is computed as:

$$
\begin{aligned}
L_{a b}^{H L I^{(k)}}(\theta) & \\
= & \sum_{1}^{k} \sum_{j \in \mathcal{P}(k)}\left(L_{a b}^{F E M}\left(\theta_{j}\right)-\sum_{i \in \mathcal{P}^{(k-1)}} \mathcal{L}_{i}^{k-1}\left(\theta_{j}\right)\right) \cdot \mathcal{L}_{j}^{k}(\theta)+\sum_{j \in \mathcal{P}(0)} L_{a b}^{F E M}\left(\theta_{j}\right) \\
& \mathcal{L}_{j}(\theta)
\end{aligned}
$$

As stated in Section 2.1.2, for the static eccentricity fault the computation of $\left[L_{s s}\right]$ and $\left[L_{s r}\right]$ only requires the results of the FEM simulations feeding each stator phase for the first 36 rotor positions which means that the rotor has to be moved between 0 to $2 \pi / 1008 \cdot 36=\pi / 14$ rad. Hence the parametric space is defined as $[0, \pi / 14] \mathrm{rad}$. In the same way, to compute the coupling parameters associated to rotor phases (or rotor bars) one must feed a rotor phase and performing the magnetostatic FEM simulation for the first 504 positions which implies the parametric space $[0, \pi]$ rad. Tables 2 and 3 show the set of GCL points, i.e. the rotor positions $\theta$, for the different hierarchical levels $k$ in which the magneto-static FEM simulation should be performed. Thereafter, the polynomial basis according to (11) is computed with the results of FEM simulations with the rotor placed in the positions of Tables 2 and 3 and used to compute the coupling parameters between stator phases $\left[L_{s s}\right]$, between stator phases and rotor bars $\left[L_{s r}\right]$ and between rotor bars $\left[L_{r r}\right]$ respectively.

However, the computation of the polynomial basis for the case of coupling parameters between rotor bars requires a deeper analysis. Fig. 4 shows the coupling parameters between the 1st rotor bar with itself based on FEM simulations. It can be seen as these coupling parameters can be computed as a sum of two functions. Due to the slot effect, there is a first function with $\pi / 14$ period associated to the movement of a rotor bar through a stator slot. On the other hand, due to the static eccentricity fault, there is a second function with a $2 \pi$ period that behaves similarly to the air-gap width depending on the rotor position. It can be seen that the higher fault severity the higher the amplitude of this second function. Therefore, as the fault severity degree increases this $2 \pi$ period function becomes more prominent whereas for low severity degrees the slot effect is more prominent. Thus, both effects have to be considered to accurately compute the

Table 2

Set of the GCL points in the parametric space $[0, \pi / 14]$ to compute $\left[L_{s s}\right],\left[L_{s r}\right]$ and include the slot effect in $\left[L_{r r}\right]$.

\begin{tabular}{cccc}
\hline Level $(k)$ & Rotor position $\theta\left(P^{(k)}\right)$ rad & Level $(k)$ & Rotor position $\theta\left(P^{(k)}\right) \mathrm{rad}$ \\
\hline 0 & 0 & 3 & 0.0085 \\
0 & $\pi / 14=0.2244$ & 3 & 0.0693 \\
1 & $\pi / 28=0.1122$ & 3 & 0.1551 \\
2 & 0.0329 & 3 & 0.2159 \\
2 & 0.1915 & & \\
\hline
\end{tabular}


Table 3

Set of the GCL points in the parametric space $[0, \pi]$ to include the static eccentricity effect in $\mathrm{t} \quad$ [Lh $\mathrm{A}$ computation.

\begin{tabular}{cccc}
\hline Level $(k)$ & Rotor position $\theta\left(P^{(k)}\right)$ rad & Level $(k)$ & Rotor position $\theta\left(P^{(k)}\right) \mathrm{rad}$ \\
\hline 0 & 0 & 3 & 0.1196 \\
0 & $\pi$ & 3 & 0.9697 \\
1 & $\pi / 2$ & 3 & 2.1719 \\
2 & 0.4601 & 3 & 3.0220 \\
2 & 2.6815 & & \\
\hline
\end{tabular}

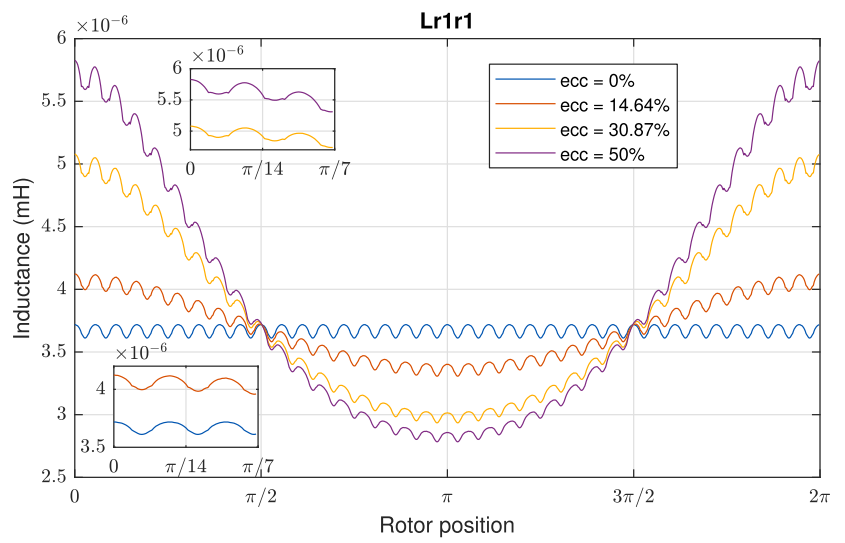

Fig. 4. Coupling parameters between rotor bar 1 and itself for different rotor positions for four different degrees of static eccentricity fault. These coupling parameters have been computed through FEM simulations. Zoom of the coupling parameters have been included to show the effect of the slots.

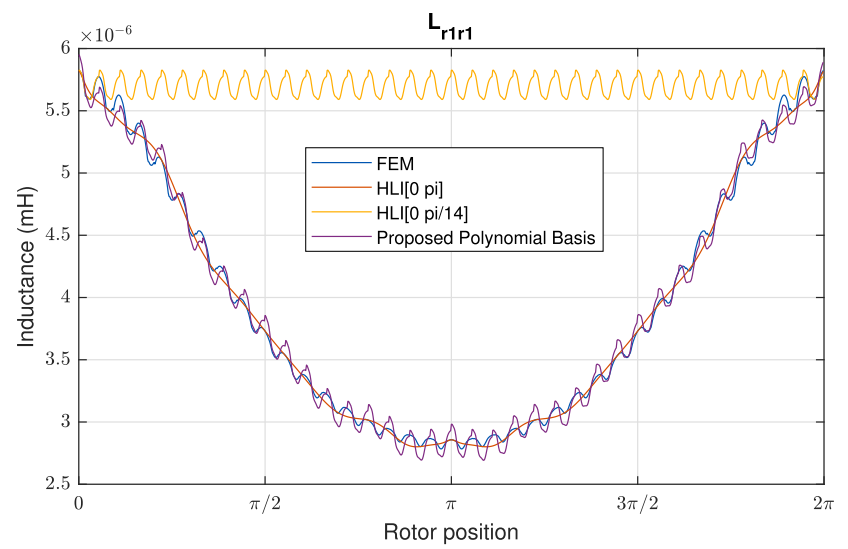

Fig. 5. Comparison of coupling parameters between rotor bar 1 and itself for different rotor position and for a static eccentricity of 50\% computed with FEM, HLI considering the parametric space $[0, \pi]$ rad, HLI considering the parametric space $[0, \pi / 14]$ rad and with the polynomial basis proposed as a combination of the HLI obtained with both parametric spaces $[0, \pi / 14] \operatorname{rad}$ and $[0, \pi] \operatorname{rad}$.

coupling parameters between rotor bars. This fact suggests that two parametric spaces should be considered to obtain the polynomial basis to compute the rotor rotor coupling parameters: $[0, \pi / 14]$ rad to include the slot effect, $[0, \pi]$ rad to consider the effect of the static eccentricity fault. To better illustrate this fact, Fig. 5 compares the coupling parameters obtained with FEM analysis between those obtained with the polynomial basis obtained in: the $[0, \pi]$ rad parametric space, the $[0, \pi / 14]$ rad parametric space or as proposed, i.e., as a combination of both polynomial basis. It can be seen, that the proposed polynomial combination yields better results and reflects more accurately the coupling parameters if FEM analysis was performed but a lower cost.

\subsection{Results}

To check the effectiveness of the proposed method different polynomial basis have been computed considering the different hierarchical levels 0 to 3 of Tables 2 and 3. Considering the polynomial basis obtained for each hierarchical level $k$, the coupling parameter matrix, $[L]$, has been computed and compared with the results obtainedusing the FEM simulations and the procedure depicted in Fig. 3 particularized for the case of static eccentricity fault described in Section 2.1.2.

Fig. 6 shows the evolution of three elements of the coupling parameters matrix $[L]$ depending on the rotor position: between the stator phase 1 with itself $\left[L_{S_{1} s_{1}}\right]$, between the stator phase 1 and the rotor bar 1 $\left[L_{s_{1} r_{1}}\right]$ and between the rotor bar 1 with itself $\left[L_{r_{1} r_{1}}\right]$ for three different levels of static eccentricity (0\% (Healthy), $14.64 \%$ and 50\%). This figure compares the results of computing the coupling parameters using the full FEM analysis with the results obtained with the proposed method considering different hierarchical levels $k$ to compute the polynomial basis $L^{H L I^{(k)}}$ according to (11). It must be highlighted that the proposed method uses,for each hierarchical level $k$, just the results of the FEM simulations for the rotor positions in the set of the GCL points shown in Tables 2 and 3.

To check the accuracy of the proposed method the error between the coupling parameters computed with FEM $L^{F E M}$ and with the proposed method $L^{H L I^{(k)}}$ for each hierarchical level $k$ is computed as:

$\operatorname{error}(\%)=$ mean $\left|\frac{L^{F E M}(\theta)-L^{H L I}(k)}{(\theta)}\right| \cdot 100$

Fig. 6 bottom shows the mean error obtained in the computation of the three coupling parameters $\left(L_{s_{1} s_{1}}, L_{s_{1} r_{1}}, L_{r_{1} r_{1}}\right)$ for the three different levels of static eccentricity depending on the hierarchical level $k$ considered. It can be seen that, as the hierarchical level $k$ increases the accuracy improves due to the more FEM simulations used to obtain the polynomial basis. However, it can be also seen that the level $k=2$ could be enough to compute the coupling parameters. For this hierarchical level the error is less than the $2 \%$ and the use of more points (higher hierarchical level) does not significantly improves the accuracy but adds more computational effort.

This, in turn, implies that just with results of the FEM simulations for the rotor placed in the five positions for the hierarchical levels $k=0$ to $k=2$ of Table 2 is enough to compute the coupling parameters matrix. Additionally, for the rotor-rotor coupling parameters the positions corresponding to levels $k=0$ to $k=2$ of Table 3 are also required to build the two polynomial basis aforementioned.

Table 4 shows the computational costs in terms of FEM simulations, computation time and memory resources for data storage for the generic case (according to the procedure shown in Section 2.1), the generic case particularized for the static eccentricity fault (according the procedure shown in Section 2.1.2) and for the proposed method (depicted in Section 3). In view of the results, it must be highlighted that the proposed method computes the coupling parameters 24.48 times faster and requires just one $4.09 \%$ of the memory resources for the generic case particularized for the static eccentricity fault (Table 4: 3rd column) and keeping the accuracy better than $2 \%$.

\section{Simulation}

Once the coupling parameters are computed, they are used in the model shown in Fig. 2. The model has been implemented in the HIL OP4500 whose main characteristics are detailed in the Appendix A. The HIL runs the model in real time and the stator currents as well as other signals such as the speed can be sampled in real time through the analog outputs. Hence, as shown in Fig. 7 these signals can be used to test fault diagnosis techniques implemented in embedded devices, to train and test CM systems based on artificial neural network (ANN) or to be acquired through a digital oscilloscope and processed offline in a 


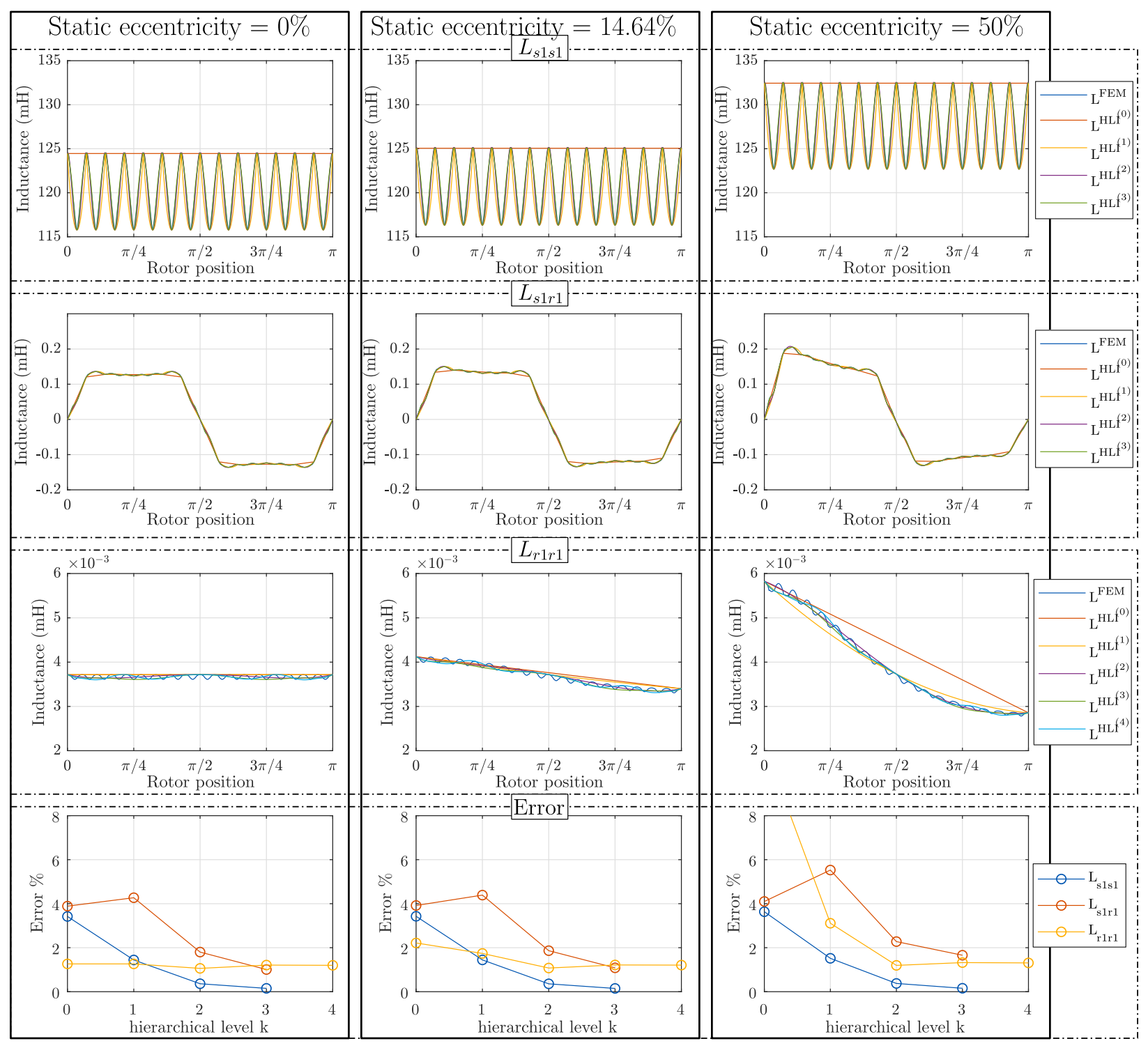

Fig. 6. The figure shows the coupling parameters computed with FEM analysis and with the proposed method, $L^{H L I^{k}}$ where $k$ is the hierarchical level of the polynomial basis defined in (11) and computed with the GCL points of Tables 2 and 3. From top to bottom: coupling parameters between stator phase 1 with itself, $L_{s_{1} s_{1}}$, between stator phase 1 and rotor bar $1, L_{s_{1} r_{1}}$ and between rotor bar 1 with itself $L_{r_{1} r_{1}}$ for three different degrees of severity of static eccentricity fault (0\% Healthy, $14.64 \%$ and 50\%). Finally, the bottom figure shows the error, computed according (12), committed in the computation of each coupling parameters with the proposed method depending on the hierarchical level $k$ of the polynomial basis used.

pc system to develop other fault diagnosis techniques.

\subsection{Fault diagnosis using the proposed IM running in a HIL simulator}

Transient based fault diagnosis methods have attracted a rising interested due to their reliability. They can be used to detect faults in wide variety of working conditions such as oscillating loads, inverterfed motors with changes of speed, start-up transients, supply variations, etc. Hence, they allow to reduce misdiagnoses generally associated with steady state fault diagnosis techniques due to situations that could be confused with faults [1,35-39]. Indeed, the current analysis during the IM start-up transient is widely used because the evolution of the slip is well-known (from 1 to $\simeq 0$ ) and it is possible to identify the different patterns followed by the fault harmonic components. Moreover, it does not require the speed measurement.

Table 4

Computational effort and memory resources needed to obtain the coupling parameters of a faulty IM model in a generic case (1st column), particularizing for the static eccentricity fault (2nd column) and with the proposed method (3rd column).

\begin{tabular}{lccc}
\hline & Generic case & Static eccentricity & Proposed method \\
\hline $\mathrm{n}^{\text {er }}$ FEM simulations & 17,136 & 612 & 25 \\
Computation time & 11 days $21 \mathrm{~h} 36 \mathrm{~min}$ & $10 \mathrm{~h} 12 \mathrm{~min}$ & $25 \mathrm{~min}$ \\
Data storage & $376.52 \mathrm{~GB}$ & $13.45 \mathrm{~GB}$ & $0.55 \mathrm{~GB}$ \\
\hline
\end{tabular}




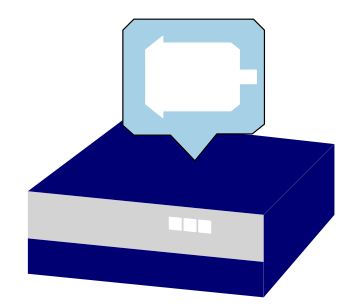

REAL TIME SIMULATOR OP 4500

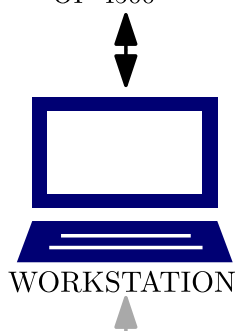

Fig. 7. The hybrid FEM- analytical model developed is transferred to the real time simulator. The real-time signals needed (stator currents, speed) are connected to the analogue outputs of the HIL and used for different fault diagnosis purposes: to develop, train and test either AI based or continuous on-line (embedded devices) fault diagnosis systems. On the other hand, these signals can also be acquired through digital oscilloscope or directly transferred to the pc-station for further processing.

\subsection{Evolution of the static eccentricity fault harmonic components}

For the static eccentricity fault the frequencies of the harmonic components are defined by (1). Considering the specific parameters of the motor defined in Table 1 the evolution of the fault harmonic components during the start-up transient can be easily computed as the slip evolves from 1 to $\simeq 0$. Consequently, for the lower side harmonic (LSH), $v=-1$, its frequency evolves from $50 \mathrm{~Hz}$ to $\simeq 650 \mathrm{~Hz}$. Similarly. the upper side harmonic (USH), $v=1$, evolves from $50 \mathrm{~Hz}$ to $\simeq 750 \mathrm{~Hz}$. Besides, the amplitude of the LSH remains almost constant regardless the fault severity degree while in case of the USH its amplitude increases as the severity degree increases [40].

Therefore, to check the usability of the parameters computed with the proposed method, in terms of fault diagnosis purposes, three different simulations, with three degrees of static eccentricity (Healthy, $30 \%$ and $50 \%$ ) fault have been performed. The simulations have been performed during the start-up transient and the currents have been sampled during $2 \mathrm{~s}$ from the analogue outputs of the HIL, OPAL OP4500, at a sampling frequency of $10 \mathrm{kHz}$. The main objective is to compare the results obtained from the model using the coupling parameters computed with the proposed method and with the coupling parameters fully computed with the traditional FEM analysis following the procedure shown in Fig. 3. For fault diagnosis purposes, these results have to be compared in terms of the evolution of the static eccentricity fault harmonic components during the start-up transient. Fig. 8 shows the spectrogram of the stator current for the simulated machine with the coupling parameters computed with the prosed method and compared with those obtained using the coupling parameters computed with FEM. As can be seen, the LSH appears regardless the machine status and it is a reason why this component is used for speed prediction in some control drives. On the other hand, the USH component appears only when the machine is under faulty conditions and it becomes more clear as the severity degree increases. As can be seen, these results reinforce the validity of the proposed method as it obtains the same results, with minor errors, as those obtained with the traditional FEM analysis.

\section{Experimental validation}

The proposed method has been validated with a commercial $1.1 \mathrm{~kW}$ IM (whose main characteristics are the same as the simulated model and shown in the Table 1) to observe the presence of the fault harmonic components. The experimental set up is show in Fig. 9. To achieve longer startup transients the IM has been feed to reduced voltage through an auto-transformer and no external load has been used. To introduce the static eccentricity fault the hood fastening holes have been slightly enlarged to achieve a small tolerance in the positioning of the rotor axis as shown in Fig. 10. The stator currents have been sampled using a digital oscilloscope during $10 \mathrm{~s}$ at a sampling rate of $10 \mathrm{kHz}$.

The stator current spectrogram for the IM in the same conditions as bought (considered as healthy) and for the IM faulty machine are shown in Fig. 11 where the LSH and USH harmonic components are highlighted. As can be seen, for the healthy machine the USH is also visible as IMs are not ideal and each IM has inherent eccentricity that should be lower than $10 \%$ as stated in [10]. However, it should be highlighted that, as the fault severity degree increases (faulty machine) the amplitude of the USH also increases as shown in the simulation results and confirmed by this experimental validation. Therefore, tracking the evolution of this fault harmonic component could be an useful tool for condition monitoring of IMs.

\section{Conclusions}

This paper proposes the sparse identification to reduce the computational effort required to compute the coupling parameters of a FEManalytical model of a faulty IMs. The, proposed method achieves a significant improvement in the computing time and in the memory resources while keeping a good accuracy. In fact, it is 24.48 times faster computing the coupling parameters and requires just the $4.09 \%$ of the memory resources than a full FEM analysis. Besides, the actual error between the coupling parameters computed with FEM and with the proposed method is less than $2 \%$. The proposed method takes into account the special effects of the geometry of the induction motor such as the slot effect or the winding effect. Moreover it can consider the variations the air-gap width due to a fault.

In this paper, the proposed method has been illustrated for the static eccentricity fault with different fault degrees from incipient levels or even inherent levels of the machine to more severe scenarios which would help in the aim of correlating the amplitude of the fault harmonic components with the fault severity degree. After that, the models have been tested with the coupling parameters computed and the fault diagnosis results (fault harmonic components) have been compared with those obtained with the models where the coupling parameters have been fully computed with FEM software. Besides, these results have been confirmed by experimental validation. Following the same reasoning the method could be extended to other types of faults or even to simultaneous faults.

Finally, it must be highlighted that the proposed method would have a major impact in the fast development of IM models with different types of fault and/or with different degrees of severity of a given fault. Therefore, the method will favour the development of fault diagnosis systems, especially on-line fault diagnosis system and AI based systems as it will help not only to cover a wide variety of scenarios (machines, degrees of severity and types of fault, working conditions) but also in establishing thresholds for the early detection of a given fault.

\section{Declaration of Competing Interest}

The authors declare that they have no known competing financial interests or personal relationships that could have appeared to 

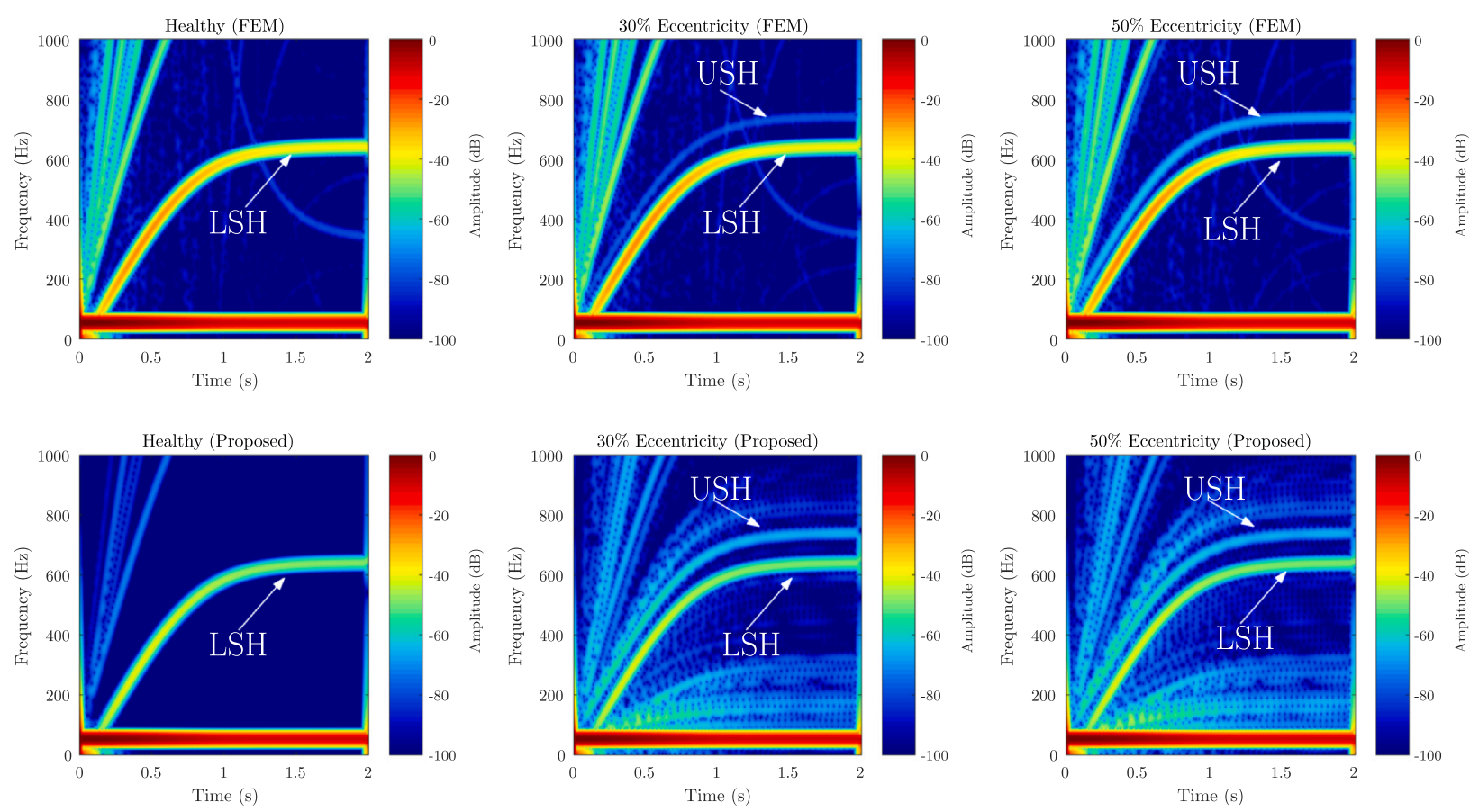

Fig. 8. Stator current spectrogram for the three different levels of static eccentricity using the proposed method (HLI $I^{(2)}$ ) and FEM software to compute the coupling parameters of the hybrid FEM-analytical model. The fault harmonic components LSH and USH due to static eccentricity fault have been highlighted for both methods.

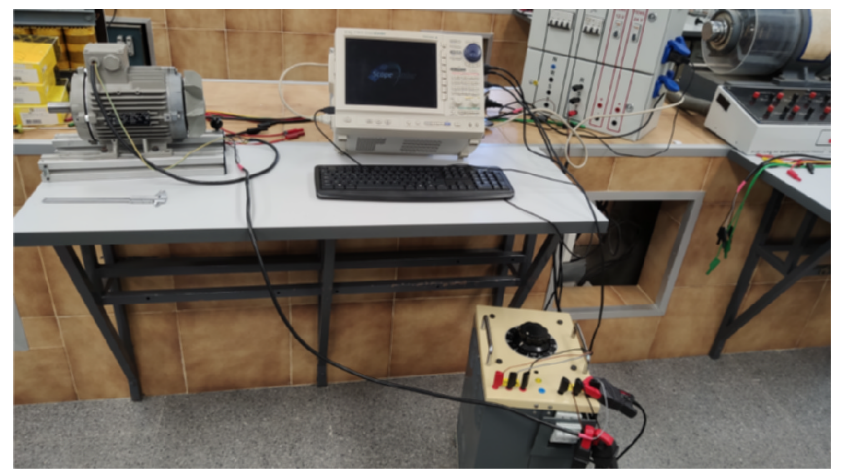

Fig. 9. Test bed used for the experimental validation. The motor is fed through an autotransformer to a low voltage to achieve a longer start-up transient. The stator currents have been sampled using digital oscilloscope with the aid of current clamps.

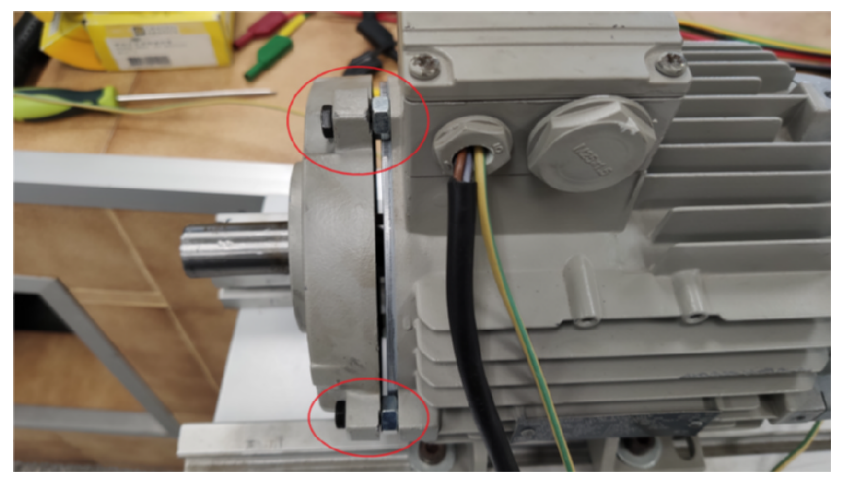

Fig. 10. Detail of the hood fasten holes drilled to introduce the static eccentricity fault.
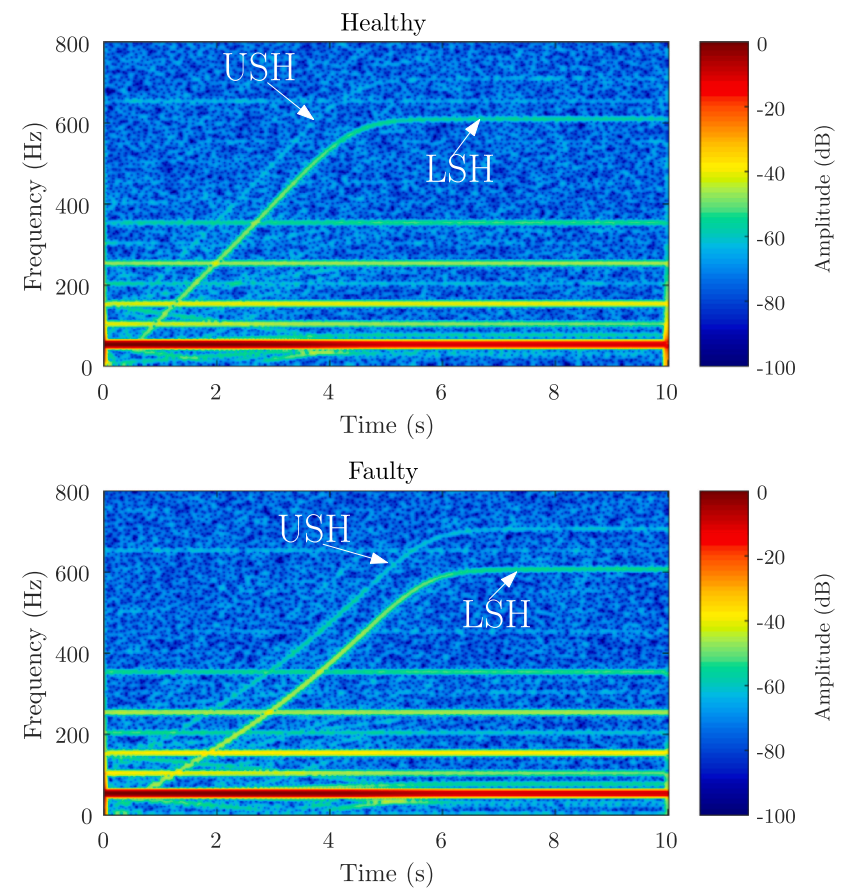

Fig. 11. Stator current spectrogram for the IM in the same conditions as it was bought, i.e. in healthy conditions (top) and with the static eccentricity fault (bottom). It can be seen that the start up transients lasts $5 \mathrm{~s}$ and can be see both USH and LSH fault harmonic components. It must be highlighted as the USH increases its amplitude as the fault severity degree increases as shown in the simulation results.

influence the work reported in this paper.

\section{Acknowledgement}

This work was supported by the Spanish "Ministerio de Ciencia, Innovación y Universidades (MCIU)", the "Agencia Estatal de 
Investigación (AEI)" and the "Fondo Europeo de Desarrollo Regional (FEDER)" in the framework of the "Proyectos I+D+i - Retos Investigación 2018", project reference RTI2018- 102175-B-I00 (MCIU/ AEI/FEDER, UE).

This work was supported by the Spanish "Ministerio de Educación, cultura y Deporte" in the framework of the "Programa Estatal de Promoción del Talento y su Empleabilidad en I $+D+i$, Subprograma Estatal de Movilidad, del Plan Estatal de Investigación Científica y Técnica y de Innovación 2013-2016" in the subframework "Estancias de movilidad en el extranjero José Castillejo para jóvenes doctores".

\section{Appendix A. OPAL 4500 main features}

Real- time target: 4 INTEL processor cores $3.3 \mathrm{GHz}$ (only 1 core activated). Solid state disk: $125 \mathrm{~Gb}$. Memory RAM: $4 \mathrm{~Gb}$. Real-time operating system: Linux RedHat. Xilinx Kintex 7 FPGA (326.000 Logic cells and 840 DSP slice). Sampling Rate: $200 \mathrm{MHz} .96$ User Inputs/ Outputs (I/O): 16 analog inputs and 16 analog outputs, 24 digital inputs and 24 digital outputs, 8 RS422 digital inputs and 8 RS422 digital outputs.

\section{References}

[1] Karmakar S, Chattopadhyay S, Mitra M, Sengupta S. Induction motor fault diagnosis: General discussion and research scope. Induction Motor Fault Diagnosis. Springer; 2016. p. 153-8.

[2] Bellini A, Filippetti F, Tassoni C, Capolino G-A. Advances in diagnostic techniques for induction machines. IEEE Trans Industr Electron 2008;55(12):4109-26.

[3] Faiz J, Ebrahimi B, Sharifian M. Finite element transient analysis of an on-load three-phase squirrel-cage induction motor with static eccentricity. Electromagnetics 2007;27(4):207-27.

[4] Salem SB, Salah M, Touti W, Bacha K, Chaari A. Stray Flux analysis for monitoring eccentricity faults in induction motors: Experimental study. 2017 International conference on control, automation and diagnosis (ICCAD). 2017. p. 292-7.

[5] Salah M, Bacha K, Chaari A. An improved spectral analysis of the stray flux component for the detection of air-gap irregularities in squirrel cage motors. ISA Trans 2014;53(3):816-26.

[6] Bessous N, Zouzou SE, Sbaa S, Bentrah W. A comparative study between the MCSA, DWT and the vibration analysis methods to diagnose the dynamic eccentricity fault in induction motors. In: 2017 6th International Conference on Systems and Control (ICSC); 2017. p. 414-21.

[7] Siddiqui KM, Sahay K, Giri V. Health monitoring and fault diagnosis in induction motor-a review. Int J Adv Res Electr Electron Instrument Eng 2014:3(1):6549-65.

[8] Faiz J, Moosavi S. Eccentricity fault detection-from induction machines to DFIG-a review. Renew Sustain Energy Rev 2016;55:169-79.

[9] Oumaamar MEK, Maouche Y, Boucherma M, Khezzar A. Static air-gap eccentricity fault diagnosis using rotor slot harmonics in line neutral voltage of three-phase squirrel cage induction motor. Mech Syst Sig Process 2017;84:584-97.

[10] Thomson WT, Barbour A. On-line current monitoring and application of a finite element method to predict the level of static airgap eccentricity in three-phase induction motors. IEEE Trans Energy Convers 1998;13(4):347-57.

[11] Liang J, Qiu Y, Zhao M, Kang S, Lu H. The modeling and numerical simulations of wind turbine generation system with free vortex method and simulink. Energy Convers Manage 2015;103:762-77.

[12] Shukla RD, Tripathi RK. Isolated wind power supply system using double-fed induction generator for remote areas. Energy Convers Manage 2015;96:473-89.

[13] Chen X, Hu J, Chen K, Peng Z. Modeling of electromagnetic torque considering saturation and magnetic field harmonics in permanent magnet synchronous motor for HEV. Simul Model Pract Theory 2016;66:212-25.

[14] Pantea A, Yazidi A, Betin F, Taherzadeh M, Carrière S, Henao H, et al. Six-phase induction machine model for electrical fault simulation using the circuit-oriented method. IEEE Trans Industr Electron 2016;63(1):494-503.

[15] Naderi P, Taheri A. Slot numbering and distributed winding effects analysis on the torque/current spectrum of three-phase wound-rotor induction machine using discrete modeling method. Electr Power Compon Syst 2015;43(15):1717-26.

[16] Chang H-C, Lin S-C, Kuo C-C, Hsieh C-F. Induction motor diagnostic system based on electrical detection method and fuzzy algorithm. Int J Fuzzy Syst
2016;18(5):732-40.

[17] Dong G, Ojo O. Efficiency optimizing control of induction motor using natural variables. IEEE Trans Industr Electron 2006;53(6):1791-8.

[18] Gradev S, Reuss J, Herzog H-G. A general voltage-behind-reactance formulation of a multivoltage N 3-phase hybrid-excited synchronous machine. IEEE Trans Energy Convers 2016;31(4):1452-61.

[19] Tavana NR, Dinavahi V. Real-time nonlinear magnetic equivalent circuit model of induction machine on FPGA for hardware-in-the-loop simulation. IEEE Trans Energy Convers 2016;31(2):520-30.

[20] Duan F, Živanović R, Al-Sarawi S, Mba D. Induction motor parameter estimation using sparse grid optimization algorithm. IEEE Trans Industr Inf 2016;12(4):1453-61.

[21] Ghoggal A, Zouzou SE, Razik H, Sahraoui M, Khezzar A. An improved model of induction motors for diagnosis purposes-Slot skewing effect and air-gap eccentricity faults. Energy Convers Manage 2009;50(5):1336-47.

[22] Martinez J, Belahcen A, Detoni J. A 2D magnetic and 3D mechanical coupled finite element model for the study of the dynamic vibrations in the stator of induction motors. Mech Syst Sig Process 2016;66:640-56.

[23] Gu B-G. Offline Interturn fault diagnosis method for induction motors by impedance analysis. IEEE Trans Industr Electron 2017;65(7):5913-20.

[24] Aguiar VP, Pontes RS, Neto TRF, Souza KN. Comparison of FEA field models combined with analytical method to determine the performance characteristics of high efficiency induction motors, in. Power electronics conference and 1st southern power electronics conference (COBEP/SPEC), 2015 IEEE 13th Brazilian. IEEE; 2015. p. 1-6.

[25] Ling Z, Zhou L, Guo S, Zhang Y. Equivalent circuit parameters calculation of induction motor by finite element analysis. IEEE Trans Magn 2014:50(2):833-6.

[26] Alvarez-Gonzalez F, Griffo A, Sen B, Wang J. Real-time hardware-in-the-loop simulation of permanent-magnet synchronous motor drives under stator faults. IEEE Trans Industr Electron 2017;64(9):6960-9.

[27] Poveda-Lerma A, Sapena-Bano A, Garcı-Lameiras A, Riera-Guasp M., MartinezRoman J. Induction machine model for fault diagnosis using hardware in the loop and finite element analysis. In: Congress on numerical methods in engineering CMN2017, vol. 3; 2017. p. 5.

[28] Borzacchiello D, Aguado JV, Chinesta F. Non-intrusive sparse subspace learning for parametrized problems. Arch Comput Methods Eng 2019;26:303-26.

[29] Sapena Bano A, Chinesta F, Pineda-Sanchez M, Aguado J, Borzacchiello D, PuchePanadero R. Induction machine model with finite element accuracy for condition monitoring running in real time using hardware in the loop system. Int J Electr Power Energy Syst 2019;111:315-24.

[30] Horen Y, Strajnikov P, Kuperman A. Simple mechanical parameters identification of induction machine using voltage sensor only. Energy Convers Manage 2015;92:60-6.

[31] Chatterjee A, Chatterjee D. An improved excitation control technique of three-phase induction machine operating as dual winding generator for micro-wind domestic application. Energy Convers Manage 2015;98:98-106.

[32] Sapena-Bano A, Martinez-Roman J, Puche-Panadero R, Pineda-Sanchez M, PerezCruz J, Riera-Guasp M. Induction machine model with space harmonics for fault diagnosis based on the convolution theorem. Int J Electr Power Energy Syst 2018;100:463-81.

[33] Lange E, Henrotte F, Hameyer K. An efficient field-circuit coupling based on a temporary linearization of FE electrical machine models. IEEE Trans Magn 2009;45(3):1258-61.

[34] Peherstorfer B, Zimmer S, Bungartz H-J. Model reduction with the reduced basis method and sparse grids. Sparse grids and applications. Springer; 2012. p. 223-42.

[35] Siddiqui KM, Sahay K, Giri V, Gothwal N. Diagnosis of airgap eccentricity fault in the inverter driven induction motor drives by transformative techniques. Perspect Sci 2016:8:127-31.

[36] Burriel Valencia J, Puche-Panadero R, Martinez-Roman J, Sapena-Bano A, PinedaSanchez M. Short-frequency fourier transform for fault diagnosis of induction machines working in transient regime. IEEE Trans Instrum Meas 2017;66(3):432-40.

[37] Burriel Valencia J, Puche-Panadero R, Martinez-Roman J, Sapena-Bano A, RieraGuasp M, Pineda-Sanchez M. Multi-band frequency window for time-frequency fault diagnosis of induction machines. Energies 2019;12(17):3361.

[38] Burriel Valencia J, Puche-Panadero R, Martinez-Roman J, Sapena-Bano A, PinedaSanchez M. Fault diagnosis of induction machines in a transient regime using current sensors with an optimized slepian window. Sensors 2018;18(1):146.

[39] Sapena Bano A, Pineda-Sanchez M, Puche-Panadero R, Martinez-Roman J, Matic D. Fault diagnosis of rotating rlectrical machines in transient regime using a single stator current's FFT. IEEE Trans Instrum Meas 2015;64(11):3137-46.

[40] Sapena-Bano A, Riera-Guasp M, Martinez-Roman J, Pineda-Sanchez M, PuchePanadero R, Perez-Cruz J. fem-analytical hybrid model for real time simulation of ims under static eccentricity fault. In: 2019 IEEE 12th international symposium on diagnostics for electrical machines, power electronics and drives (SDEMPED). 